\title{
Clinical Events Sponsor Defined Identifier
}

National Cancer Institute

\section{Source}

National Cancer Institute. Clinical Events Sponsor Defined Identifier. NCI Thesaurus. Code C87856.

One or more sponsor defined characters used to identify, name, or characterize the clinical event. 